\title{
Load Dependence of Power Outage Statistics
}

\author{
SOUmyajyoti Biswas ${ }^{1,2}$ and LuCAS GOEHRING ${ }^{3}$ \\ 1 Max Planck Institute for Dynamics and Self-Organization - Am Fassberg 77, 370r7 Göttingen, Germany \\ 2 WW8-Materials Simulation, Department of Materials Science, Friedrich-Alexander-Universität Erlangen-Nürnberg, \\ Dr.-Mack-Str. 77, 90762 Fürth, Germany \\ 3 School of Science and Technology, Nottingham Trent University, Clifton Lane, Nottingham NG11 8 NS, UK
}

\author{
PACS 45.70.Ht - Avalanches \\ PACS 89.75.Da - Systems obeying scaling laws \\ PACS 64.60.aq - Networks
}

\begin{abstract}
Dynamics of power outages remain an unpredictable hazard in spite of expensive consequences. While the operations of the components of power grids are well understood, the emergent complexity due to their interconnections give rise to intermittent outages, and power-law statistics. Here we demonstrate that there are additional patterns in the outage size distributions that indicate the proximity of a grid to a catastrophic failure point. Specifically, analysis of the data for the U.S. between 2002-2017 shows a significant anti-correlation between the exponent value of the power-law outage size distribution and the load carried by the grid. The observation is surprisingly similar to dependencies noted for failure dynamics in other multi-component complex systems such as sheared granulates and earthquake statistics, albeit under much different physical conditions. This inspires a generic threshold-activated model, simulated in realistic network topologies, which could successfully reproduce the exponent variation in similar range. Given sufficient data, the methods proposed here can be used to indicate proximity to failure points and forecast probabilities of major blackouts with a non-intrusive measurement of intermittent grid outages.
\end{abstract}

Introduction. - A secure supply of electrical power is a vital component of a prosperous society, and any interruptions or outages can have catastrophic consequences, as witnessed on many occasions [1]. Characterisations of power grid instabilities and outages, therefore, have been active topics of research for decades in both the physics and engineering communities (see e.g. $[2-8]$ ). An operating power grid, particularly near its permissible level of capacity, can suffer from large outages triggered by small initial fluctuations or disturbances [9]. For example, a software failure in an early warning management system [10], a tree falling on a line [11] or overloading by users [12] caused blackouts affecting, respectively, about 55, 56 and 620 million people. Such an amplified response to a smallscale perturbation is a prominent signature of the systemwide correlations which develop near a critical point.

Another clear example of correlated response in power grids is the distribution function of outage sizes, as measured, for example, by the number of customers left unserved during an outage. While random and independent failure events would result in an exponentially decaying distribution of outage sizes, in reality the probability $p(S)$ of an outage of size $S$ has a power law tail, $p(S) \sim S^{-\alpha}$, reflecting the relatively higher probabilities of large outages [14] due to causally connected cascades of outage events. A rank-plot of event sizes is often used to characterise such a power-law distribution $[2,13]$, due to its relationship with the cumulative probability distribution (see Eqs. (1-3)). Statistical analyses of grid outages, particularly those demonstrating the universality of the exponent value, $\alpha$, across different countries [14], has led to the recognition of the dynamics of power outages as a case of self-organised criticality (SOC) $[15,16]$. Indeed, a connected set of objects (e.g. high-voltage power lines) having finite failure thresholds, coupled with a drive (customer demand) and dissipation (load unserved) is suitable for showing emergent collective behaviour and a selforganised critical state. As such, outage statistics are reminiscent of the Gutenberg-Richter-like law that describes the event (or avalanche) size distributions of earthquake statistics [17], acoustic emissions in stressed brittle solids [18], sheared granular media [19], creep rupture in 
disordered material [20] and so on.

While generally such avalanche statistics may have a scale-free size distribution, their exponent is also commonly seen to vary with the 'load' borne by a system. The nature of this load depends on the relevant drive, e.g. tectonic stress for earthquakes, or compressive stress for laboratory fracture. Specifically, if only the events occurring at a higher load are sampled, the magnitude of the exponent $\alpha$ is frequently smaller than what is obtained for events occurring at a lower load. Such behaviour was first noticed in sheared rocks [21], where $\alpha$ decreases linearly with differential stress. Subsequently, it has been observed for the acoustic emissions preceding cliff failure [22], for earthquakes [23-25] and sheared granulates [19]. For example, although the magnitudes of earthquakes worldwide follow a universal scaling, in some places or times $\alpha$ tends to be lower, signalling a higher risk of large earthquakes [26]. Recently, much advantage has been made of this insight, to generate risk-maps of earthquake activity, and to identify potential danger spots in areas like Sumatra [27] or California [28].

Here we show that there is a lowering of the size distribution exponent with increased load, i.e. customer demand, for power outage statistics. Using the publicly available data (see [29]) for outages in the U.S. between 2002-2017, we found significant differences in the size distributions of events occurring in the night or day, over which time the total power usage changes by about a third. Indeed, there is a systematic anti-correlation of the exponent value with the load on the grid, as measured for different hours of the day and different months of the year. Such changes are also observed for smaller regions, where they may be indicative of the relative risks of outages.

The similarities of these observations with other driven dissipative systems also encourages us to explore them through a generic threshold-activated model, without the operational complexities of the power grids but preserving a realistic network topologies. We find that such a minimal model is capable of reproducing the load-dependence features of power outage statistics, suggesting that it is, indeed, a robust feature of the near-critical dynamics of power grids.

Outages in the U.S. grid. - The size distribution of power outages in the U.S. has been studied, both in terms of the power left unserved and the number of customers affected $[2,9,13,30]$. In fact, these quantities vary almost co-linearly, except in a few instances involving load shed affecting e.g. one customer, such as may be the case for a large industrial facility. The two metrics also show power-law size distributions, with exponent values that are identical to within the relevant error bars [30]. For example, the cumulative distribution function for the number of customers affected during blackouts is reported to follow a power law, with exponents variously estimated in the range from 0.8 to $1.3[2,13,30]$. Similar studies for outages in Sweden [31], Norway [32], New Zealand [33]

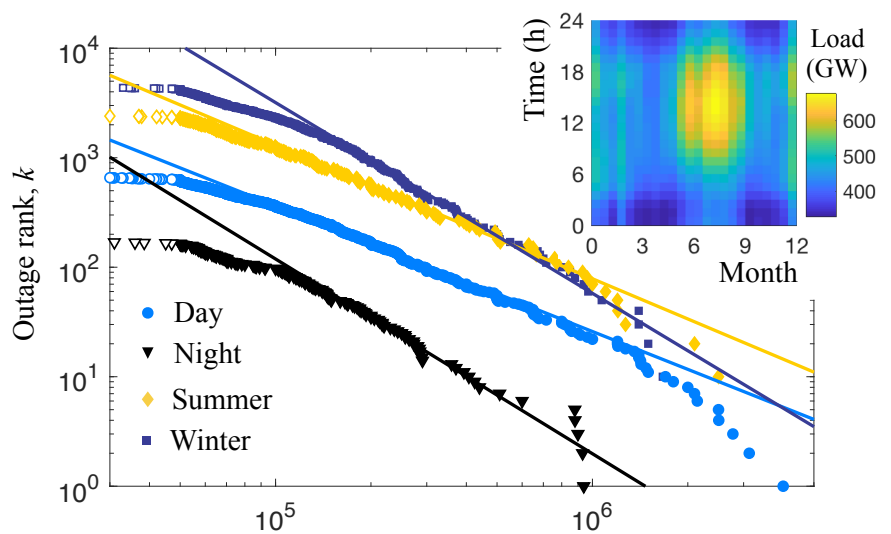

(a)

Size of outage, $S_{k}$ (customers affected)

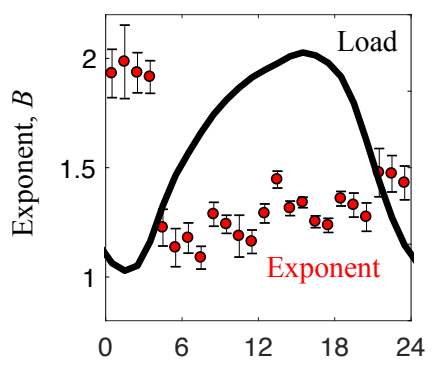

(b)

Time of Day (h)

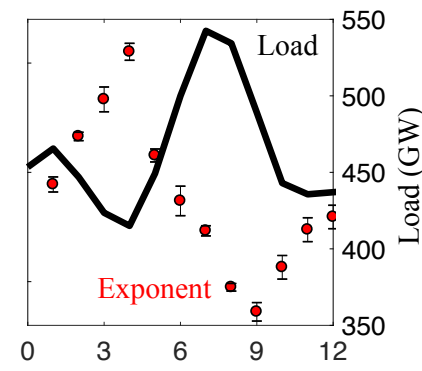

(c) Month of Year

Fig. 1: The sizes of large-scale outages in the U.S. follow a power-law distribution, whose exponent changes with the load on the grid at the time of outage. (a) We demonstrate this by dividing the 1193 publicly reported outages from 2002-2017 into those occurring during the day and night, or the summer and off-peak/winter periods. The lower load cases (night and winter) show steeper power-law distributions than the higher load cases (day and summer). Events below size 50,000 are not required to be publicly reported, and are distinguished as open circles. The seasonal plots are also shifted 10x up the $\mathrm{y}$ axis, to aid visibility. The inset shows the national electricity consumption at different times and months, for 2016. This robust anti-correlation between the load and exponent can be seen if the data are further subdivided according to (b) month (3-month rolling average) or (c) time of day (3-hour rolling average).

and China [34] also reveal scale-free size distributions of power outages. Outage events have also been fit with nonpower law scalings [30]. While the lower end of the data can be well-fit with other distributions, the tail of extreme events is usually found to be well-approximated by a power law $[2,13,30-34]$, and we follow this procedure here. We will show that the exponent value of such a distribution depends on the load carried by the power grid, at the time of failure.

Data are collected from the U.S. Energy Information Administration website (see [29]), which lists outage events affecting more than 50,000 consumers, or resulting in a load shedding of more than $300 \mathrm{MW}$, as well as the hourly electricity demand. There are 1193 reported outages in the period 2002-2017 (inclusive) affecting known, 
non-zero numbers of customers, which we consider. Outage times are recorded in the local time zone. The load values used are for 2016, and reported nationally using the Pacific time zone as a reference. Hourly load data was taken from the 1st and 15th of every month, avoiding weekends and holidays (specifically, using January 4th/15th; May 2nd/13th; and October 3rd/14th), when load patterns are different. Daily average loads were collected throughout the year. Load averages and standard deviations were calculated from these data for each window of hours or months used.

For fitting the outage size distributions, we use rank plots. The events $S$ can be arranged in the descending order of their sizes:

$$
S_{1} \geq S_{2} \geq S_{3} \ldots \geq S_{n}
$$

The $k^{t h}$ ranked element has size $S_{k}$. For events with a probability distribution $p(S)$, the number of events having size greater than or equal to $S_{k}$ is

$$
\int_{S_{k}}^{\infty} p(S) d S=k .
$$

If $p(S) \sim S^{-\alpha}$, then

$$
k \sim S_{k}^{(1-\alpha)}=S_{k}^{-B} .
$$

The ranked data was fit in two ways. First, a maximum likelihood estimator (MLE) method [13] was used to measure the exponent $B$, and an event size cutoff. Second, we applied least-squares fits to the data. The least squares fits were performed for both the raw data, and data binned to have equal widths (a factor of 2 ) on a log-scale. For this an a priori cutoff is required. This is taken as 50,000 (the requirement for reporting) or above when there are other signatures of under-reporting (e.g. kink at 100,000 consumers in Fig. 2(b)). Error estimates for least squares fits were checked by repeating fits on randomly subsampled data sets (100 trials on half-sampled data); the resulting spread in exponents is consistent with the stated fit errors. While the precise exponent values depend on the method of fitting used, they show same trends with load. Complete sets of fits for both methods are given in the online supplementary material; least-squares fits for the binned data are used in the manuscript and manuscript figures.

In Fig. 1(a) the rank plots, or cumulative size distributions, are given for the subsets of power outages occurring respectively during the day (08:00-20:00, local times), night (22:00-04:00), summer (July-August), or winter (October-May). These periods were chosen to correspond with times of peak and off-peak loads, as measured by the national electricity demand during 2016, and shown in the inset to Fig. 1(a).

A power law fit of the whole data set gives an exponent $B=1.30 \pm 0.02$, which is consistent with previous reports $[2,13,30]$. However, following a day/night division these outages split into a shallower daytime distribution with
$B=1.15 \pm 0.03$ and a steeper nighttime distribution with $B=1.78 \pm 0.02$. While it is known that there are fewer outages at night, than in the day [30], our result shows that those outages that do occur at night are generally also much less severe. Similarly, if the data are split seasonally, we find an exponent of $B=1.22 \pm 0.04$ in the months of peak summer usage, but an exponent of $B=1.74 \pm 0.04$ during the off-peak winter months.

To further demonstrate the significant relationship between the load on the grid and the exponent value of the outage size distribution, we have considered outages in rolling three-hour time windows. Fig. 1(b) shows the variations of the exponent $B$ and the load for different hours of the day. Similarly, Fig. 1(c) shows the loads and exponents for different months of the year, using a three-month rolling window. The fits for all windows used are provided in the online supplementary aterial. For both cases an anti-correlation between the load and the fitted exponent value can be seen. Both the Student's t-test and Spearman's rank-order correlation reject the null hypothesis of no correlation, at more than $99 \%$ confidence. Thus, like a variety of other driven disordered systems [19, 21-24], we find that a higher load is associated with a smaller $B$ value, and hence a more extreme distribution of events.

So far we have demonstrated a temporal variation of the outage size distribution exponent over the entire U.S. power grid. However, in order to show the potential for identifying any vulnerable areas or dangerous hot-spots on a grid, it is important to consider such load dependence on different spatial scales, as well. The U.S. grid is divided between regional reliability councils, or RRC's (see Ref. [29] for source of maps). We chose the three regions with the greatest number of recorded outages and fit the outage size distributions of events from the day and night, in each region. The results, shown in Fig. 2, show that load-dependent variations can be established on smaller regions of a grid. In the online supplementary material we also analyse the whole data set from each RRC, and show that there are significant variations in exponent across the U.S., ranging from $B=1.67$ in RFC (Northeast), to 0.70 in FRCC (Florida). In order to probe to even finer scales, such as is done with $B$-value maps of earthquake risks, one would need access to a larger volume of fine-grained data. In practice, this would mean records substantially below the required thresholds of public reporting, in the U.S.

Modeling outages. - We explore the loaddependence of power grids further via a network model, with different topologies and loading conditions. There are many approaches to modelling power grid dynamics, including examples of networks obeying circuit laws $[6,35-37]$, sometimes incorporating phase information $[3,38-40]$ leading to the examination of the transient behaviour during the outage (e.g. propagation of desynchronisation), as well as more abstract models [41,42], alongside a large literature on failures in complex networks in general (see e.g. [43-46]). Here we use a model of the 


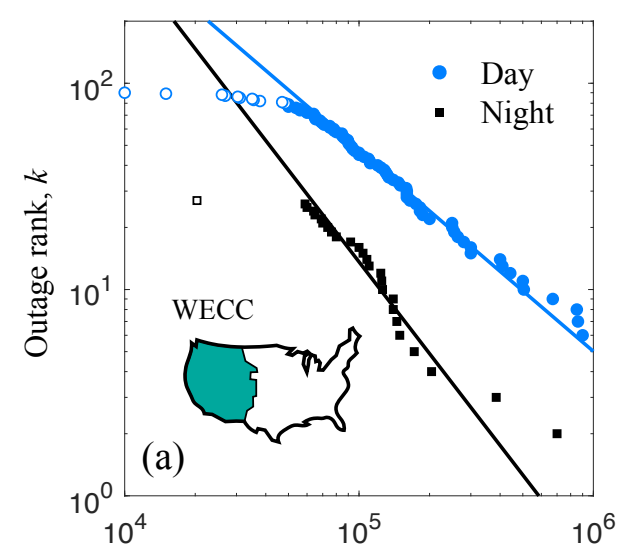

Size of outage, $S_{k}$ (customers affected)

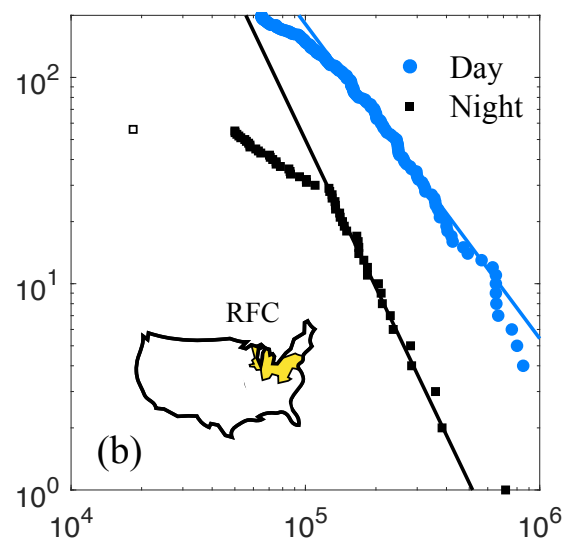

Size of outage, $S_{k}$ (customers affected)

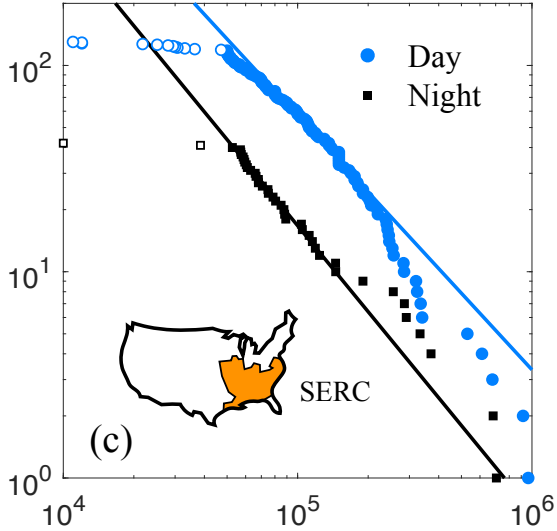

Size of outage, $S_{k}$ (customers affected)

Fig. 2: Day-night variations in outage size distributions are also seen regionally. Outages in the U.S. are divided according to their governing Regional Reliability Council (RRC). Shown are the rank size distributions for the three RRC's with the most reported events, (a) WECC (149 events), (b) RFC (315 events) and (c) SERC (182 events), divided between day (10:00 - 20:00, local time) and night (00:00-06:00). In each case the nighttime outage distribution is steeper $(B=1.39,1.48$, and 2.39 , for (a)-(c), respectively) than the day-time distribution $(B=1.23,0.97$, and 1.52$)$, indicating that outage events are generally more severe at higher-load times.

power grid similar to that studied in Refs. [47-50] where we assume a wide separation of time scales between internal relaxation of the system and external drive. The model demonstrates how the observed U.S. outage data match generic features of the load-dependence of outage statistics.

Specifically, we consider a set of elements, or nodes, having finite failure thresholds. The elements are either connected to each other in a topology simulating the Western Interconnection of the U.S. grid [51], or by a regular lattice. The thresholds $\sigma_{t h}^{i}$ and loads $\sigma_{l}^{i}$ of the $i^{t h}$ element are related by

$$
\sigma_{t h}^{i}=\sigma_{l}^{i}+s \epsilon_{i}
$$

We assign a random load $\sigma_{l}^{i}$ to each element, from a uniform distribution between zero and one. The second term on the right hand side provides a buffer or redundancy for the elements, with $s$ quantifying their typical level of redundancy. This ensures that the capacity of an element $\sigma_{t h}^{i}$ is always higher than its initial load. The random variables $\epsilon_{i}$ are also chosen from a uniform distribution on $[0,1]$. Therefore, on average, the network carries a fraction of load $1 /(1+s)$, measured relative to its average capacity. The values of $1 /(1+s)$ studied are given in the legend to Fig. 3.

The dynamics of the model follow from randomly choosing an element and dropping its threshold to zero, thereby triggering a failure event. This could happen due to external causes (a storm, vandalism, etc.) on a grid or be caused by a sudden surge in demand among customers. In the model this causes a node to overload, rather than its connections or links, which instead inform how the failing load spreads. The load carried by the overloaded element is now redistributed amongst the surviving elements. That may, in turn, cause some of those elements to over- load their thresholds and break, triggering an extended avalanche. We assume that there is a separation of time scales between the spread of an event through the system, and the triggers of successive events. Indeed, while the spreading of an event takes a few seconds [39], successive triggering events could be days, weeks or further apart. Therefore, the full load of a failed element is redistributed across its neighbours, which are then tested for stability. If a completely isolated node fails (i.e. it has no remaining unbroken neighbours), its load is not redistributed, but is instead lost; this would represent an area of the grid for which there remains no possible route to deliver power. An outage is thus modelled as the redistribution of load in successive steps following a breakdown. The size of an avalanche is then the number of elements which break, before a stable configuration is reached. After the avalanche has finished, any broken elements are restored, all elements on the network are assigned new randomly selected thresholds and loads, and the whole process is repeated.

First, we consider this failure model on the topology of the Western Interconnection, as described in Ref. [51], and containing 4941 nodes connected by 6594 links. The load sharing in this case is confined to connected neighbours, such that an avalanche can only cascade along adjacent links. Specifically, all surviving neighbours are given an equal share of the load of a failed node. The resulting avalanches are recorded over time and rank-plots are shown in Fig. 3(a) for different values of the relative stress, or excess capacity. A power-law scaling is seen in all cases, with deviations only near the system size. The exponent shows a load-dependence, decreasing from $B=1.40$ to 0.41 , as the average load increases from $40 \%$ to $53 \%$ of the average capacity.

Due to the long-range nature of the correlations de- 
veloped in a real grid, a failure at one point can start an avalanche or cascade failure at a far away location [52]. Therefore, a second version of our model allows a local disturbance to trigger a remote event directly. For this, we consider 8100 elements on a two-dimensional square lattice. Following a local failure, the load carried by the broken element is now redistributed to the entire remaining network. The fraction of load accommodated by each other point depends with its distance from the failure point, $\Delta x$, scaling as $1 /(\Delta x)^{\gamma}$. We take $\gamma=2$, in keeping with the dependence of current flow in the random fuse model [53] (e.g. around an isolated flaw in a conductive plane), but note that $\gamma$ could be varied, allowing for a transition from local to mean-field behaviour [54]. The total load is kept constant in this case as well, although for long range redistribution this ignores any dissipation from transmission loss which could occur. The resulting avalanches are recorded and rank-plots are shown in Fig. 3(b) for selected values of the relative stress. Again, the rank-plot exponent varies significantly within the range of loads studied; increasing the relative load from $50 \%$ of capacity, to $63 \%$, reduces $B$ from 2.19 to 0.89 . The range of power-law behaviour in this model is more limited, however. One possible reason for this is that the system is unrealistically resilient, due to its long-range interaction. Nevertheless, this result, from a generally constructed network, covers most of the observed temporal range of $B$ values for the U.S. grid, as well as the spatial variations seen in its different regions, and corresponds to a similar relative change in electricity demand to that occurring nationally from the day, to the night.

Discussion. - The intermittent dynamics of power grid outages have been associated with self-organised criticality for almost twenty years $[13,15]$. Characteristic signatures of this, including a scale-free size distribution of the outage sizes, are seen for outages in many different countries [30-34]. This connection of power grids with self-organised criticality enables comparisons with other similar systems, such as earthquakes [13].

Here we have shown that, as for a variety of other driven disordered systems [19,21-24], power grids have a significant load-dependence in their failure statistics. When outages are ranked by the number of people affected, their statistical distribution is a power law whose exponent is anticorrelated with electrical demand. This anti-correlation was measured for data taken from the publicly reported U.S. power outages, using various rolling 3-hour or 3month windows, and comparing to the average demand in those windows (Figs. 1 and S1-S4). The results are summarised in Fig. 4(a), and show that as the load increases from $400 \mathrm{GW}$ to $550 \mathrm{GW}$ (a $37 \%$ increase), the exponent $B$ drops from about 2 to 1 . As with earthquakes [21], this dependence with load is approximately linear. Expressed in terms of probabilities, this means that for peak load, an outage affecting a million customers is about $1 \%$ as likely as that of one affecting a hundred thousand customers,

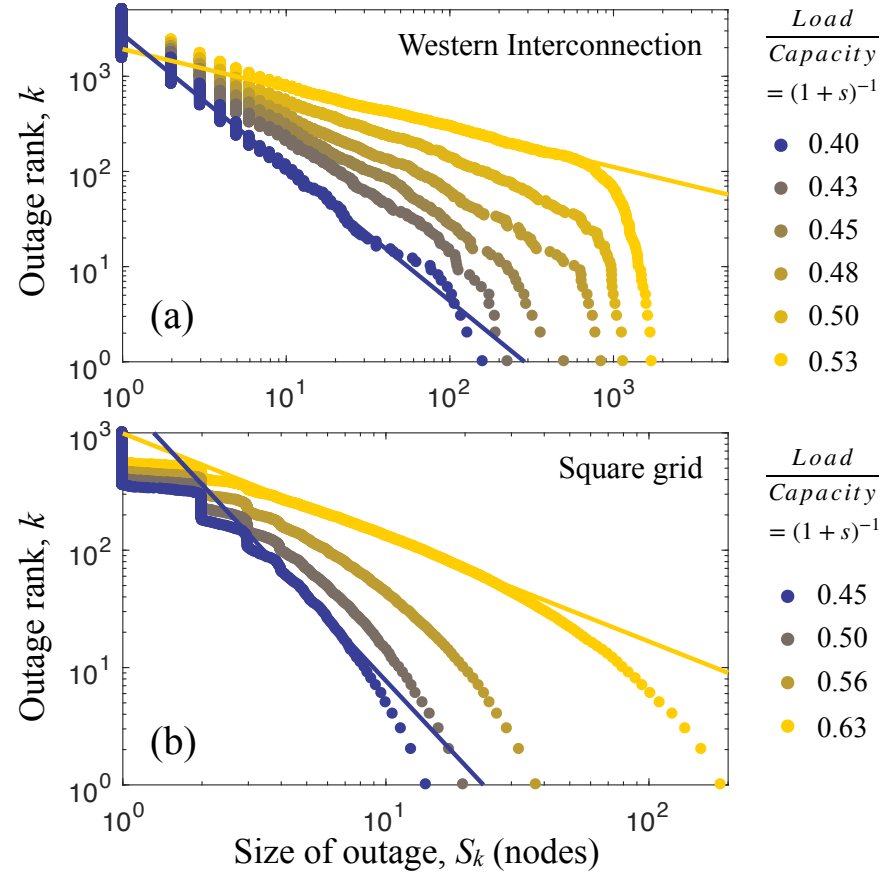

Fig. 3: Rank plots of model events demonstrate the generic nature of the load-dependence of power outage statistics. Results are shown for models with various ratios of the average load to capacity (i.e. values of $1 /(1+s)$ ) and either (a) nearestneighbour redistribution of load across a realistic topology of the Western Interconnection of the North American power grid [51] (4941 nodes), or (b) distributed load sharing on a grid with regularly spaced elements $(90 \times 90=8100$ nodes $)$. In all cases, the fitted exponents are shown in Fig. 4, and compare well to the range of exponents observed in the real U.S. grid.

while for low loading conditions, it is only about $0.1 \%$ as likely. These statistics are independent of the absolute number of outages, but are only strengthened by noting that there are fewer outages at night, than day [30].

By analysing different sub-regions of the U.S. grid, corresponding to the regional reliability councils, we have also shown (Figs. 2 and S5) that variations in $B$ can be measured locally, and display the same day/night variation as the national grid. This demonstrates the practicality of mapping variations of $B$ as an estimator of outage risk, as is now becoming routine for earthquake risk [26-28].

Given the universal nature of the anti-correlation between load and avalanche size distribution exponent, this effect should be manifested in a generic thresholdactivated process without having to consider the details of the specific system. The underlying topologies are a relevant parameter in determining universality and should, of course, influence the exact exponent values, but nevertheless preserve the anti-correlation qualitatively. This expectation was confirmed by exploring the load dependence of power grid outages in a minimal model that considers a grid as a collection of fragile nodes, which pass their demand on to other elements if they fail. Models were considered with nearest-neighbour interactions on a 

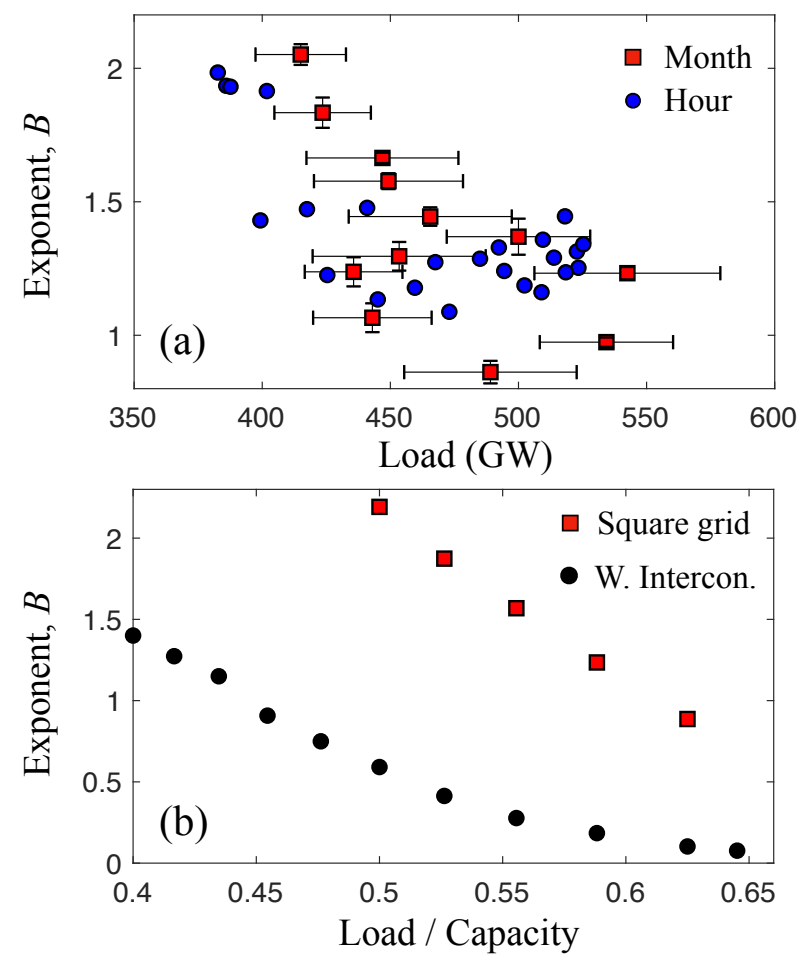

Fig. 4: Comparison of power outage statistics and loaddependence, showing the measured exponent, $B$, for outage size distributions in various situations. (a) For the U.S. grid, at different times of the day, and different months of the year, the data (from Fig. 1) demonstrate the anti-correlation of $B$ and load. Representative error bars are shown on the monthly data, and also show the standard deviation in the load during that time. (b) Modelled outage statistics, in various situations, show similar results, and explore a similar range of exponents as the load is varied.

topology following the Western Interconnection [51] in the U.S., and with long-range redistributions on a regular lattice. The nearest-neighbour model is particularly weak, as one point failing will have a high chance of taking its neighbour with it, and so on. The long-range model is, in contrast, overly strong, with a long-distance spreading of load, and hence less chance of concentrating it on a weak element. Nonetheless, as summarised in Fig. 4(b), both models showed load-dependencies, and a substantial overlap in the observed $B$ values of the real U.S. grid, for comparable changes in relative loading. A further relevant parameter in determining the exponent values could be a correlation in the drive. In the present context, nonGaussian deviations in the transmission frequencies could provide such a correlated drive, as it relates to the load imbalance in the system [55]. This could be an interesting future direction of the present study.

Conclusions. - We have demonstrated that the distribution of outage sizes in power grids depends on the load on the grid at the time of outage. Specifically, there is an almost linear decay of the exponent value $B$ of the outage size distribution with the load on the grid, a result similar to that found in earthquake size distributions. Therefore, if outage data were available with sufficient completeness, i.e. good spatial resolution and including smaller events, then a statistically significant map could be drawn for the exponent value of the size distribution of outage events in different places (as is becoming increasingly common for earthquake risk [26-28]. The spatial variation of the exponent values would then signify the relative risks of the major outages, mirroring the health of the grid. Such a risk-map would then allow for the implementation of more effective risk management and mitigation strategies to support a resilient and robust long-term power grid design.

$$
* * *
$$

SB acknowledges support from Alexander von Humboldt foundation during part of the study.

\section{REFERENCES}

[1] Andersson G., Donalck P., Farmer R., Hatziargyriou N., Kamwa I., Kundur P.,Martins N.,Paserba J., Pourbeik P., Sanchez-Gasca J., Schulz R., Stankovic A., Taylor C. and Vittal V., IEEE Trans. Power Syst., 20 (2005) 1922.

[2] Carreras B. A., Newman D. E. and Dobson I., IeEe Transactions on Power Systems, 31 (2016) 4406.

[3] Witthaut D. and Timme M., New J. Phys., 14 (2012) 083036

[4] Pahwa S.,Scoglio C. and Scala A., Sci. Rep., 4 (2014) 3694.

[5] Brummitt C. D.,D'Souza R. M. and Leicht E. A., Proc. Nat. Acad. Sci., 109 (2012) E680.

[6] Simonsen I., Buzna L., Peters K., Bornholdt S. and Helbing D., Phys. Rev. Lett., 100 (2008) 218701.

[7] Vaiman M., Bell K., Chen Y., Chowdhury B., DobSON I., Hines P.,Papic M.,Miller S. and Zhang P., IEEE Trans. Power Systems, 27 (2012) 631.

[8] Ji C., Wei Y., Mei H., Calzada J., Carey M., Church S., Hayes T., Nugent B., Stella G., Wallace M., White J. and Wilcox R., Nature Energy, 1 (2016) 16052.

[9] Yang Y., Nishikawa T. and Motter A. E., Science, 358 (2017) eean3184.

[10] U.S.-Canada Power System Outage Task Force (2004), Final report on the August 14, 2003 Blackout in the United States and Canada: Causes and Recommendations, available online at www.nerc.com.

[11] Final Report of the Investigation Committee on the 28th September 2003 Blackout in Italy, UCTE 2004 (available online at: http:www.rae.groldcasesC13italyUCTE_rept.pdf)

[12] Romero J. J., IEEE Spectrum, 49 (2012) 11.

[13] Clauset A., Shalizi C. R. and Newman M. E. J., SIAM Review, 51 (2009) 661.

[14] Dobson I., Carreras B. A., Lynch V. E. and Newman D. E., Chaos, 17 (2007) 026103.

[15] Carreras B. A., Newman D. E. and Dobson I., Initial evidence for self-organized criticality in electric power sys- 
tem blackouts, in Proc. 33rd Hawai Int. Conf. System Sci., Jan. 2000.

[16] BAK P., How nature works: the science of self.organized criticality (Springer-Verlag, New York) 1996.

[17] Gutenberg B. and Richter C. F., Bull. Seismol. Soc. Am., 46 (1956) 105.

[18] Main I. G., Kun F. and Bell A. F., Crackling Noise in Digital and Real RocksImplications for Forecasting Catastrophic Failure in Porous Granular Media. In: Salje E., Saxena A., Planes A. (eds) Avalanches in Functional Materials and Geophysics. Understanding Complex Systems. Springer, Cham (2017).

[19] Hatano T., Narteau C. and Shebalin P., Sci. Rep., 5 (2015) 12280.

[20] Castellanos D. F. and Zaiser M., Phys. Rev. Lett., 121 (2018) 125501.

[21] Scholz C. H., Bull. Seism. Soc. Am., 58 (1968) 399.

[22] Amitrano D., Grasso J. R. and Senfaute G., Geophys. Res. Lett., 32 (2005) L08314.

[23] Schorlemmer D., Wiemer S. and Wyss M., Nature, 437 (2005) 539.

[24] Scholz C. H., Geophys. Res. Lett., 42 (2015) 1399.

[25] Rivière J., Lv Z., Johnson P. A. and Maroneb C., Earth Planetary Sci. Lett., 20 (2018) 407.

[26] Nanjo K. Z., Hirata N., Obara K. and Kasahara K., Geophys. Res. Lett., 39 (2012) L20304.

[27] Nuannin P., Kulhanek O. and Persson L., J. Asian Earth Sci., 61 (2012) 237.

[28] Tormann T., Wiemer S., Mignan A., J. Geophs. Res. Solid Earth, 119, (2014) 2029.

[29] Data on U.S. Power outages reported monthly at: https://www.eia.gov/electricity/monthly/. Data on hourly electricity demand is reported at: https://www.eia.gov/realtime_grid/\#/summary/demand. The maps of the Regional Reliability Councils can be found in https://www.epa.gov/energy/north-americanreliability-corporation-nerc-region-representational-map.

[30] Hines P., Apt J. and Talukdar S., Energy Policy, 37 (2009) 5249.

[31] Holmgren $\AA$ and Molin S., J. Infrastruct. Syst., 12 (2006) 243.

[32] Bakke J. Ø. H., Hansen A. and Kertész J., EPL, 76 (2006) 717.

[33] Ancell G., Edwards C., Krichtal V.,, Is a large blackout of the New Zealand power system inevitable?, Electricity Engineers Association 2005 Conference: Implementing New Zealand's Energy Options, Aukland, New Zealand, June 2005.

[34] Weng X., Hong Y., Xue A. and Mei S., Journal of Control Theory and Applications, 4 (2006) 235.

[35] J. Chen, J. S. Thorp, I. Dobson, Int. J. Electr. Power Energy Syst., 27 (2005) 318.

[36] Rios M. A., Kirschen D. S., Jawayeera D., Nedic D. P. and Allen R. N., IEEE Trans. Power Syst., 17 (2002) 543.

[37] Kirschen D. S.,Jawayeera D., Nedic D. P. and Allan R. N., IEEE Trans. Power Syst., 19 (2004) 1650.

[38] Yang Y.,Nishikawa T., Motter A. E., Phys. Rev. Lett., 118 (2017) 048301.

[39] Schäfer B., Witthaut D., Timme M., Latora V., Nat. Comm., 9 (2018) 1975.

[40] Dewenter T., Hartmann A. K., New J. Phys., 17
(2015) 015005.

[41] DeMarco C. L., IEEE Control Syst. Mag., 21 (2001) 40.

[42] Stubna M. D. and Fowler J., Int. J. Bifurcation Chaos Appl. Sci. Eng., 13 (2003) 237.

[43] Boccaletti S., Latora V., Moreno Y., Chavez M. and Hwanga D. -U., Phys. Rep., 424 (2006) 175.

[44] Buldyrev S., Parshani R.,Paul G., Stanley H. E. and HaVlin S., Nature, 464 (2010) 1025.

[45] Goh K. I.,Kahng B. and Kim D., Phys. Rev. Lett., 87 (2001) 278701.

[46] Panzieri S. and Setola R., Int. J. Crit. Infrastruct., 4 (2008) 63.

[47] Pahwa S., Hodges A., Scoglio C. and Wood S., Topological analysis of the power grid and mittigation strategies against cascading failures, In Systems Conference, 4th Annual IEEE, (2010) 272.

[48] Yagan O., Phys. Rev. E, 91 (2015) 062811.

[49] Zhang Y. and Yagan O., Sci. Rep., 6 (2016) 27625.

[50] Biswas S. and Goenring L., Phil. Trans. R. Soc. A, 377 (2019) 20170388

[51] Watts D. J. and Strogatz S. H., Nature, 393 (1998) 440.

[52] Kosterev D. N., Taylor C. W. and Mittelstadt W., IEEE Trans. Power Syst., 14 (1999) 967.

[53] de Arcangelis L., Redner S. and Herrmann H. J., J. Physique Lett., 46 (1985) L585.

[54] Biswas S. and Goenring L., New J. Phys., 18 (2016) 103048.

[55] Schäfer B., Beck C., Aihara K., Witthaut D. Timme M, Nat. Energy, 3 (2018) 119. 